MODELING, IDENTIFICATION AND CONTROL, 2005, vOL. 26, No. 4, 201-219

doi:10.4173/mic.2005.4.2

\title{
A Comparative Study of Actuator Configurations for Satellite Attitude Control
}

\author{
RAYMOND KRISTIANSEN*, OLAV EGELAND $\dagger$ and \\ PER JOHAN NICKLASSON*
}

Keywords: Reaction wheels, magnetic torquers, controllability, satellite attitude control

\begin{abstract}
In this paper a controllability study of different actuator configurations consisting of magnetic torquers, reaction wheels and a gravity boom is presented. The theoretical analysis is performed with use of controllability gramians, and simulation results with the different configurations are presented and compared regarding settling time and power consumption to substantiate the theoretical analysis. A reference model is also introduced to show how the power consumption can be lowered to the same magnitude as when magnetic torquers are used, without degrading the satellite response significantly.
\end{abstract}

\section{Introduction}

\subsection{Background}

The orientation of a satellite in space, described relative to some other object or system, is known as the attitude of the satellite. The attitude may be changing with respect to time. Some satellites are spinning around a major axis to provide directional stabilization around this axis. These satellites are well suited for operations requiring directional attitude control, such as RF-antennas and deep space monitoring. Alternatively, a satellite can use three-axis attitude control to direct itself towards any given location is space. This solution is typically used for Earth or deep space imaging applications. To be able to control the attitude of the satellite, it must be equipped with actuators that can produce angular torque. Several types of actuators exist today, and the most common solutions used for small satellites are gyroscopic torquing and magnetic torquing.

Gyroscopic torquing is a phenomenon where an internal gyro or rotor is accelerated to apply angular torque. For this purpose, it is possible to use reaction wheels which nominally maintain zero momentum but change speed to apply torques about their spin axis. When the reaction wheels are in motion, they will also provide a stabilizing effect on the satellite, since spinning bodies are known to be gyroscopically stiff. This is an advantage since the satellite will be more robust towards external disturbances, but a problem arise when the satellite orientation must be altered for some reason, because it then requires more power to rotate it about its axis.

Magnetic torquers, or magnetorquers, have been used for attitude control of satellites since the mid-sixties, when Harold Perkel created a three-axis attitude control system

\footnotetext{
*Department of Space Technology, Narvik University College, Norway. Norway

†Department of Engineering Cybernetics, Norwegian University of Science and Technology,
} 
with one fixed-gimbal momentum wheel and magnetic torquers for controlling transverse momentum components (Kaplan, 1976). A magnetic torquer produces a magnetic dipole when current flows either way in the loop. The magnitude of this dipole is proportional to the ampere-turns and the area enclosed by the coil. A satellite carrying such magnetic torquers, will have the possibility to influence its orientation by applying current to these torquers, and in this way interact with the magnetic field surrounding the Earth.

The advantage of using reaction wheels, as compared to magnetic actuators, is the possibility of controlling the satellite independently of its geographical location and altitude. The disadvantages lie primarily in expenses, the moving parts and the weight. Magnetic torquers are inexpensive, light and contain no moving parts, and are thus less vulnerable to failure. Reaction wheels introduce nonlinearities such as friction and stiction in the wheel bearings, saturation in the wheel angular velocity, and possible misalignments of the wheels with the principal axis of the satellite. They also cost and weigh a lot more.

The major obstacle in magnetic actuation is that a magnetic torquer will produce maximum torque when it is directed normal to the magnetic field vector, and zero torque when it is aligned with the local magnetic field vector. The available torque is therefore dependent on the current local magnetic field vector, and independent torques on all three axes of a supposed control system using three orthogonal magnetic torquers are not achievable. Accordingly, the yaw axis of the satellite will not be controllable over the magnetic poles of the Earth, and the roll axis will lose its torque over the Equator. Since the magnetic field is also constantly changing, magnetic control becomes nonlinear and time-varying.

Wheel saturation is a major problem when reaction wheels are used as actuation. Constant external disturbances will lead to increased angular velocity, as the wheel is accelerated at a constant rate in order to compensate for the disturbance. Clearly, such effects can be sustained only up to a certain limit, until wheel saturation occurs. It is thus necessary with an extra set of actuators generating external torques in order to desaturate the wheels, and in that way dump angular momentum from the satellite. Magnetic torquers have often been used as desaturation actuators on satellites with reaction wheels. When magnetic torquers are the principal means of actuation, no such saturation problems arise.

Magnetic actuation together with a gravity boom has been utilized earlier on the Danish Orsted micro satellite, launched from California on January 8, 1999. Information on the attitude control system can be found in Bøgh et al. (1997), Bak et al. (1999), and in references therein. Also, the first Norwegian pico-satellite nCube, based on the CubeSat concept, is designed to use magnetic actuation for attitude control, as described in Riise et al. (2003) and Gravdahl et al. (2003).

Several missions have utilized combinations of reaction wheels and magnetic torquers for attitude control. The Odin mission, a Swedish science satellite project. used four reaction wheels as main actuators, where three were mounted orthogonally, and the fourth was mounted iso-angular to the three others and served as a backup. In addition, magnetic torquers were mounted on the satellite, but these were mainly used for momentum dumping (Von Schéele, 1996). Similarly, the UoSAT-12 mission by Surrey Satellite Technology Ltd. (SSTL) employed an attitude control system consisting of reaction wheels and magnetic torquers, in addition to a gravity boom (Steyn \& Hashida. 1999). 


\subsection{Contribution}

The contribution of this paper is the presentation of a controllability study of different actuator configurations consisting of magnetic torquers, reaction wheels and a gravity boom, and is a summary of the work reported in (Kristiansen, 2000). Mathematical models for satellite dynamics and kinematics are presented, together with models of the different actuators and the geomagnetic field of the Earth. The mathematical framework behind theoretical analysis of controllability based on gramians is also laid out, and results from this analysis are presented. In addition, theory on the linear quadratic (LQ) optimal controller used in the simulations is presented. A baseline actuator configuration consisting of three orthogonally mounted magnetic torquers in conjunction with a gravity boom is simulated, and the results regarding controllability and power consumption are compared to results from simulations with reaction wheels mounted on the body axes. Lastly, simulations of a satellite with three reaction wheels and no gravity boom are presented, and a reference trajectory is introduced to show how the power consumption can be lowered to the same magnitude as when magnetic torquers are used. without compromising the satellite response significantly.

The rest of the paper is organized as follows: Section 2 defines the different reference frames used and reviews the mathematical models of rigid-body dynamics and kinematics. In addition, actuator dynamics and modeling of the geomagnetic field of the Earth complements the section. Section 4 contains the controllability theory used in the theoretical analysis, accompanied by the controllability results for the different configurations. The controller design is performed in Section 3, and simulation results of the satellite with the different actuator configurations are presented in Section 5. Section 6 comprises final conclusions and remarks.

\section{Modeling}

\subsection{Coordinate frames}

The different reference frames used throughout the paper are given as follows:

Earth-Centered Inertial (ECI) Frame: This frame is denoted $\mathcal{F}_{i}$, and has its origin located in the center of the earth. Its $z$ axis is directed along the rotation axis of the earth towards the celestial north pole, the $x$ axis is directed towards the vernal equinox, and finally the direction of the $y$ axis completes a right handed orthogonal frame.

Earth-Centered Earth Fixed (ECEF) Frame: The ECEF frame has its origin similar to the ECI frame in the center of the Earth, and its $z$ axis directed towards the celestial north pole, but its axes rotates relative to the ECI frame with the same rate as the Earth spins about its center. The $x$ axis is always pointing towards the intersection of $0^{\circ}$ latitude and $0^{\circ}$ longitude. This frame is denoted $\mathcal{F}_{e}$.

North East Down (NED) Frame: This frame is denoted $\mathcal{F}_{n}$, and is defined as an earth fixed tangent plane on the surface of the Earth with the $x$ axis and $y$ axis pointing towards true north and east, respectively. The $z$ axis is pointing towards the center of the earth, and is perpendicular to the tangent plane of the Earths reference ellipsoid. The location of the NED frame relative to ECEF frame is determined by using the geocentric latitude and longitude.

Orbit Reference Frame: The orbit frame, denoted $\mathcal{F}_{o}$, has its origin located in the mass center of the satellite. The $z$ axis is pointing towards the center of the earth, and the $x$ 
axis is directed forward in the travelling direction of the satellite, tangentially to the orbit. In a circular orbit, the orbit frame rotate relative to the ECI frame with an angular velocity of approximately

$$
\omega_{o} \approx \sqrt{\frac{\mu_{g}}{r_{c}^{3}}}
$$

where $\mu_{g}$ is the Earth's gravitational coefficient and $r_{c}$ is the distance from the frame origin to the center of the earth. Satellite rotation about the $x$-, $y$ - and $z$-axis is named roll, pitch and yaw respectively.

Body Reference Frame: This frame has, similar to the orbit frame, its origin located in the satellitc center of mass, but its axes are fixed in the satellite body and coincide with the principal axes of inertia. The frame is denoted $\mathcal{F}_{b}$.

\subsection{Kinematics}

Transformation between the previously described reference frames is done by rotation matrices, members of the special orthogonal group of order three, i.e.

$$
S O(3)=\left\{\mathbf{R} \mid \mathbf{R} \in \mathbb{R}^{3 \times 3}, \mathbf{R}^{T} \mathbf{R}=\mathbf{I}, \operatorname{det} \mathbf{R}=1\right\}
$$

where $\mathbf{I}$ is the $3 \times 3$ identity matrix. A rotation matrix for a rotation $\theta$ about an arbitrary unit vector $\mathbf{k}$ can be angle-axis parameterized as

$$
\mathbf{R}_{k, \theta}=\mathbf{I}+\mathbf{S}(\mathbf{k}) \sin \theta+\mathbf{S}^{2}(\mathbf{k})(1-\cos \theta)
$$

and coordinate transformation of a vector $\mathbf{r}$ from frame $a$ to frame $b$ is written as $\mathbf{r}^{b}=\mathbf{R}_{a}^{b} \mathbf{r}^{a}$. In general, the matrix describing transformations from the orbit frame to the body frame can be described by

$$
\mathbf{R}_{o}^{b}=\left(\mathbf{c}_{1} \mathbf{c}_{2} \mathbf{c}_{3}\right)
$$

where the elements $\mathbf{c}_{i}$ are the directional cosines. The time derivative of a matrix $\mathbf{R}_{a}^{b}$ can according to Egeland \& Gravdahl (2002) be expressed as

$$
\dot{\mathbf{R}}_{b}^{a}=\mathbf{S}\left(\omega_{a b}^{a}\right) \mathbf{R}_{b}^{a}=\mathbf{R}_{b}^{a} \mathbf{S}\left(\omega_{a b}^{b}\right)
$$

where $\omega_{a b}^{b}$ is the angular velocity of frame $b$ relative to frame $a$ represented in frame $b$ and $\mathbf{S}(\cdot)$ is the cross product operator given by

$$
\mathbf{S}(\boldsymbol{\omega})=\boldsymbol{\omega} \times\left[\begin{array}{ccc}
0 & -\omega_{z} & \omega_{y} \\
\omega_{z} & 0 & -\omega_{x} \\
-\omega_{y} & \omega_{x} & 0
\end{array}\right]
$$

where $\omega=\left[\omega_{x} \omega_{y} \omega_{z}\right]^{T}$. Similar to (4), the time derivative of the directional cosines in (3) can be expressed as

$$
\dot{\mathbf{c}}_{i}=\mathbf{S}\left(\mathbf{c}_{i}\right) \omega_{o b}^{b}
$$

The rotation matrix in (2) can be expressed by an Euler parameter representation given as

$$
\mathbf{R}_{\eta, \boldsymbol{\epsilon}}=\mathbf{I}+2 \eta \mathbf{S}(\boldsymbol{\epsilon})+2 \mathbf{S}^{2}(\boldsymbol{\epsilon})
$$

where

$$
\begin{aligned}
& \eta=\cos (\theta / 2) \in \mathbb{R} \\
& \boldsymbol{\epsilon}=\mathbf{k} \sin (\theta / 2) \in \mathbb{R}^{3}
\end{aligned}
$$


are the Euler parameters, which satisfies the constraint

$$
\eta^{2}+\boldsymbol{\epsilon}^{T} \boldsymbol{\epsilon}=1
$$

A vector consisting of the Euler parameters

$$
\mathbf{q}=\left[\eta \boldsymbol{\epsilon}^{T}\right]^{\mathrm{T}}
$$

is in the following treated as a unit quaternion vector, and referred to as a quaternion. The inverse rotation is given by the complex conjugate of $\mathbf{q}$ as

$$
\overline{\mathbf{q}}=\left[\eta-\boldsymbol{\epsilon}^{T}\right]^{T}
$$

It should be noted that if $\mathbf{q}$ represents a given attitude, then $-\mathbf{q}$ represents the same attitude after a rotation of $\pm 2 \pi$ about an arbitrary axis. Hence, even though $\mathbf{q} \neq-\mathbf{q}$ mathematically, they represent the same physical attitude.

The kinematic differential equations can be found from (4) together with (6)-(7) as

$$
\begin{aligned}
& \dot{\eta}=-\frac{1}{2} \boldsymbol{\epsilon}^{T} \boldsymbol{\omega}_{o b}^{b} \\
& \dot{\boldsymbol{\epsilon}}=\frac{1}{2}[\eta \mathbf{I}+\mathbf{S}(\boldsymbol{\epsilon})] \boldsymbol{\omega}_{o b}^{b}
\end{aligned}
$$

The deviation between the current attitude $\mathbf{q}=[\eta \boldsymbol{\epsilon}]^{T}$ and the desired attitude $\mathbf{q}_{d}=\left[\eta_{d} \boldsymbol{\epsilon}_{d}\right]^{T}$ is given by the quaternion product (Egeland \& Gravdahl, 2002) as

$$
\left[\begin{array}{c}
\tilde{\eta} \\
\tilde{\boldsymbol{\epsilon}}
\end{array}\right]=\left[\begin{array}{c}
\eta_{d} \\
\boldsymbol{\epsilon}_{d}
\end{array}\right] \otimes\left[\begin{array}{c}
\eta \\
-\boldsymbol{\epsilon}
\end{array}\right]=\left[\begin{array}{c}
\eta_{d} \eta+\boldsymbol{\epsilon}_{d}^{T} \boldsymbol{\epsilon} \\
\eta_{d} \boldsymbol{\epsilon}-\eta \boldsymbol{\epsilon}_{d}-\mathbf{S}\left(\boldsymbol{\epsilon}_{d}\right) \boldsymbol{\epsilon}
\end{array}\right]
$$

\subsection{Dynamics}

With the assumptions of rigid body movement, the dynamical model of a satellite can be found from Euler's moment equation as (Sidi, 1997)

$$
\begin{aligned}
\mathbf{J} \dot{\omega}_{i b}^{b} & =-\boldsymbol{\omega}_{i b}^{b} \times\left(\mathbf{J} \omega_{i b}^{b}\right)+\tau_{d}^{b}+\tau_{a}^{b} \\
\boldsymbol{\omega}_{o b}^{b} & =\boldsymbol{\omega}_{t b}^{b}+\omega_{o} \mathbf{c}_{2}
\end{aligned}
$$

where $\mathbf{J}$ is the satellite inertia matrix, $\omega_{i b}^{b}$ is the angular velocity of the satellite body frame relative to the inertial frame and $\omega_{o b}^{b}$ is the angular velocity of the satellite body frame relative to the orbit frame, all expressed in the body frame. The parameter $\tau_{d}^{b}$ is the total disturbance torque, $\boldsymbol{\tau}_{a}^{b}$ is the actuator torque, and $\mathbf{c}_{2}$ is the directional cosine vector from (3).

\subsection{Actuator torque}

The actuator torque $\tau_{a}^{b}$ in (12) is produced by a set of orthogonally placed magnetic torquers, located on each of the $\mathscr{F}_{b}$ axes. In addition, reaction wheels are in turn added to the body axes to investigate the effect on system performance and power consumption. Accordingly, the actuator torque can be written

$$
\tau_{a}^{b}=\tau_{m}^{b}+\tau_{w}^{b}
$$

where $\tau_{m}^{b}$ and $\tau_{w}^{b}$ are the torques generated by the magnetic torquers and the reaction wheels, respectively. 
2.4.1. Geomagnetic field. Under the assumptions that only negligible electric field changes occur and that the amount of current flowing across the boundary between the Earth and the atmosphere is relatively significant, a solution for the main geomagnetic field of the earth can be obtained from the negative gradient of a scalar potential as (Campbell, 1997)

$$
\mathbf{B}=-\left[\mathbf{i} \frac{\partial V}{\partial x}+\mathbf{j} \frac{\partial V}{\partial y}+\mathbf{k} \frac{\partial V}{\partial z}\right]=-\nabla V
$$

where $\mathbf{i}, \mathbf{j}$ and $\mathbf{k}$ represents three orthogonal directions. From Maxwell's equations we know that the divergence of the field is zero, given as

$$
\nabla \mathbf{B}=\left[\frac{\partial B_{z}}{\partial x}+\frac{\partial B_{y}}{\partial y}+\frac{\partial B_{z}}{\partial z}\right]=0
$$

By inserting equation (14) into (15) we obtain the relation known as Laplace's equation

$$
\nabla^{2} V=0
$$

which implies that this potential function will be valid over a spherical surface through which current does not flow. Equation (16) can be written in spherical notation according to

$$
\frac{\partial}{\partial r}\left[r^{2} \frac{\partial V}{\partial r}\right]+\frac{1}{\sin \theta} \frac{\partial}{\partial \theta}\left[\sin \theta \frac{\partial V}{\partial \theta}\right]=-\frac{1}{\sin ^{2} \theta} \frac{\partial^{2} V}{\partial \phi^{2}}
$$

in which $r, \theta$ and $\phi$ are the geographic, earth centered coordinates of the radial distance, co-latitude ${ }^{1}$ and longitude, respectively. When the assumption that essentially all contribution to the field comes from the internal earth sources is made, the scalar potential can be expressed in spherical coordinates as

$$
V(r, \theta, \phi)=a \sum_{n=1}^{\infty}\left[\left(\frac{a}{r}\right)^{n+1} F_{n}^{i}(\phi, \theta)\right]
$$

where $a=R_{e}=6371.2 \mathrm{~km}$ is the Earth radius, $r=R_{n}=6971.2 \mathrm{~km}$ is the orbit radius and $F_{n}^{i}(\phi, \theta)$ is the Legendre polynomial of the independent variable $\theta$ that is multiplied by sine and cosine of the independent variable $\phi$. The labeling superscript $i$ indicates internal source terms of the potential functions. The Legendre polynomial $F_{n}^{i}(\phi, \theta)$ can be expressed as

$$
F_{n}^{i}=\sum_{m=0}^{n}\left[g_{n}^{m} \cos (m \phi)+h_{n}^{m} \sin (m \phi)\right] P_{n}^{m}(\theta)
$$

where $g_{n}^{m}$ and $h_{n}^{m}$ are Gaussian coefficients, and $P_{n}^{n}(\theta)$ is the Gauss function of co-latitude only. The degree $n$ in the equation has a value of one or greater, and the order $m$ is always less than or equal to $n$. With a degree $n=1$ and order $m=0,1$, the magnetic field model is a conventional dipole model. The choice of degree and order of the magnetic field depends on the desired accuracy needed, and it is desired to have a relatively good approximation of the field without requiring enormous calculating capabilities. It is argued, however, that increasing the order of the spherical harmonic model not necessarily increase the accuracy. Bak (2000) suggest that the model of order 8 serves as a better simulation model of the geomagnetic field than that of order 10 , based on the

\footnotetext{
'The co-latitude is given as $90^{\circ}$-latitude. For more information, see Campbell (1997).
} 

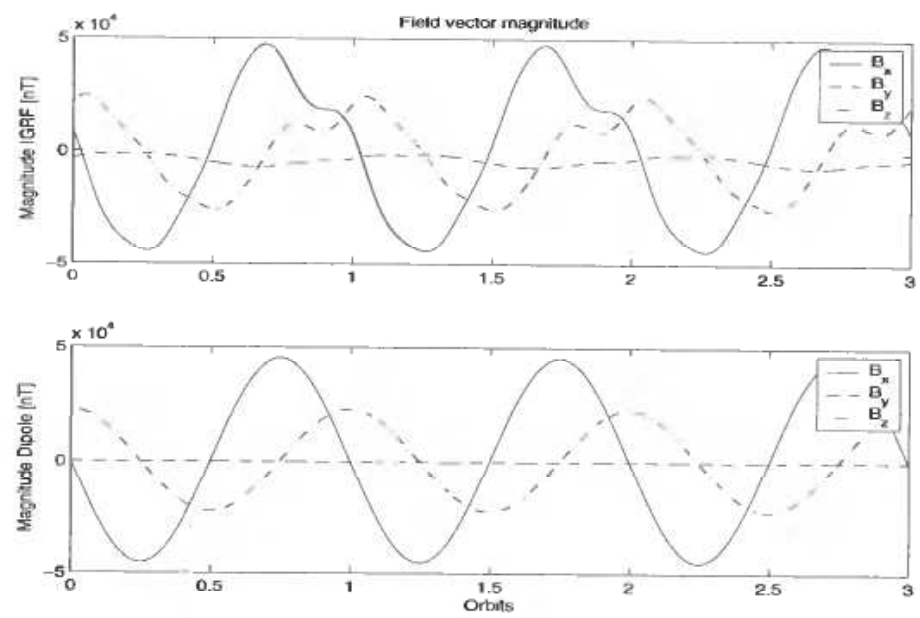

Figure 1. The magnitude of the magnetic field vectors in an IGRF model with order 10 and degree 10 (upper) and the conventional dipole model (lower).

comparison of an 8 'th order reference model and measured data from the FREJA satellite. The reference field of order 10 includes higher harmonics than that of order 8 , and thus incorporates higher frequency components of the field. It should also be pointed out that when the altitude above the Earth surface is increased, the variations become more regular, and the magnetic field resembles more and more a dipole model. A description of the field magnitude over three orbits for a conventional dipole model and a field model of order 10 and degree 10 is shown in Figure 1. From (14) and (17), the field B can be calculated as (Wertz. 1978)

$$
\begin{aligned}
& B_{r}=-\frac{\partial V}{\partial r} \\
& B_{\theta}=-\frac{1}{r} \frac{\partial V}{\partial \theta} \\
& B_{\phi}=-\frac{1}{r \sin \theta}
\end{aligned}
$$

$B_{r}$ is the outward positive radial component of the field, $B_{0}$ is the south positive coelevation component, and $B_{\phi}$ is the east positive azimuthal component. These field components can be expressed relative to an oblate Earth in the NED coordinate frame as

$$
\mathbf{B}^{n}=\left[\begin{array}{c}
-B_{\theta} \cos \varepsilon-B_{r} \sin \varepsilon \\
B_{\phi} \\
B_{\theta} \sin \varepsilon-B_{r} \cos \varepsilon
\end{array}\right]
$$

where $\varepsilon \equiv \mu_{d}-\delta<0.2^{\circ} ; \mu_{d}$ is the geodetic latitude and $\delta=90^{\circ}-\theta$ is the declination. The magnetic field vector can now be expressed in the orbit frame by using the rotation matrices between the different frames, i.e.

$$
\mathbf{B}^{o}=\left[\begin{array}{c}
B_{x}^{o} \\
B_{y}^{o} \\
B_{z}^{o}
\end{array}\right]=\mathbf{R}_{n}^{o} \mathbf{B}^{n}=\mathbf{R}_{i}^{o} \mathbf{R}_{e}^{i} \mathbf{R}_{n}^{e} \mathbf{B}^{n}
$$


The rotation matrix $\mathbf{R}_{n}^{o}$ can be written out in the form (Kristiansen, 2000)

$$
\mathbf{R}_{n}^{o}=\left[\begin{array}{ccc}
2 c \mu s \mu s^{2} \lambda & -2 c \mu c \lambda s \lambda & -1+2 c^{2} \mu s^{2} \lambda \\
-2 s \mu s \lambda c \lambda & c^{2} \lambda-s^{2} \lambda & -2 c \mu c \lambda s \lambda \\
1-2 s^{2} \mu c^{2} \lambda & 2 s \mu s \lambda c \lambda & -2 c \mu s \mu s^{2} \lambda
\end{array}\right]
$$

where $\mu$ and $\lambda$ are the geocentric lattitude and longitude, respectively, and $c \cdot$ and $s \cdot$ are the respective abbreviations of $\cos (\cdot)$ and $\sin (\cdot)$. Finally, the magnetic field can now be expressed in the body frame as

$$
\mathbf{B}^{b}=\left[\begin{array}{c}
B_{x}^{b} \\
B_{y}^{b} \\
B_{z}^{b}
\end{array}\right]=\mathbf{R}_{o}^{b} \mathbf{B}^{o}
$$

2.4.2. Magnetic torquers. The total torque generated on the satellite by the magnetic torquers can be expressed in $\mathcal{F}_{b}$ as (Marshall \& Skitek, 1990)

$$
\boldsymbol{\tau}_{m}^{b}=\mathbf{m}^{b} \times \mathbf{B}^{b}=\mathbf{S}\left(\mathbf{m}^{b}\right) \mathbf{B}^{b}
$$

where $\mathbf{m}^{b}$ is the magnetic dipole moment and $\mathbf{B}^{b}$ is the local geomagnetic field vector. The magnetic dipole moment is given by the sum of all the partial moments from the magnetic torquers

$$
\mathbf{m}^{b}=\mathbf{m}_{x}^{b}+\mathbf{m}_{y}^{b}+\mathbf{m}_{z}^{b}=\left[\begin{array}{c}
N_{x} i_{x} A_{x} \\
N_{y} i_{y} A_{y} \\
N_{z} i_{z} A_{z}
\end{array}\right]=\left[\begin{array}{c}
m_{x} \\
m_{y} \\
m_{z}
\end{array}\right]
$$

where $N_{k}$ is the number of windings in the magnetic coil on the axis in the $k$ direction, $i_{k}$ is the coil current and $A_{k}$ is the coil area.

2.4.3. Reaction Wheels. Neglecting friction and stiction in the wheel bearings, the reaction wheel equation can be written as (Kaplan, 1976)

$$
\boldsymbol{\tau}_{w}^{b}=\frac{d \mathbf{h}_{w}}{d t}+\boldsymbol{\omega}_{i b}^{b} \times \mathbf{h}_{w}=\left[\begin{array}{l}
\dot{h}_{w x}+h_{w z} \omega_{y}-h_{w y} \omega_{z} \\
\dot{h}_{w y}+h_{w x} \omega_{z}-h_{w z} \omega_{x} \\
\dot{h}_{w z}+h_{w y} \omega_{x}-h_{w x} \omega_{y}
\end{array}\right]
$$

where $\tau_{w}^{b}$ is the reaction wheel torque expressed in the body frame and $\mathbf{h}_{w}=\left[\begin{array}{lll}h_{w x} & h_{w y} & h_{w z}\end{array}\right]^{T}=\mathbf{I}_{w} \boldsymbol{\omega}_{w}$ is the total angular momentum of the wheels. Due to the principle of conservation of angular momentum, a torque rotating the reaction wheels will produce an opposite directed torque on the satellite.

\subsection{Disturbance torques}

The disturbance torques influencing on a satellite in its orbit are caused by both internal and external effects. Internal disturbances owe mostly to electromagnetic torques and fuel sloshing. External disturbances are dominated by the gravity gradient torque and 
aerodynamic drag, but also solar wind and radiation, variations in the gravitational field and collisions with meteoroids could be mentioned. These torques differ very much in magnitude, but relative to the control torques from the satellite they are small. In accordance with the discussion performed in (Kristiansen, 2000), all disturbance torques are neglected in the following, except for the dominating gravity gradient torque, which can be expressed as

$$
\tau_{g}^{b}=3 \omega_{0}^{2} \mathbf{c}_{3} \times\left(\mathbf{J c}_{3}\right)
$$

This gravity gradient torque is exploited by using a gravity boom for passive stabilization of the satellite in the Earths gravity field. The gravity boom is in this paper modeled as an ideal rigid body. However, it should be noted that the gravity boom is likely to introduce oscillatory disturbances. The amplitude and frequency of these oscillation is dependent on its construction.

\section{Controller design}

\subsection{Linearized model}

A linearized state space model of the satellite dynamical model (12) and the attitude kinematic equations in (9) and (10) can be written as

$$
\dot{\mathbf{x}}(t)=\mathbf{A x}(t)+\mathbf{B}(t) \mathbf{u}(t)
$$

where the state vector $\mathbf{x}$ and the torque vector $\mathbf{u}$ are given as

$$
\begin{aligned}
\mathbf{x} & =\left[\begin{array}{llllll}
\boldsymbol{\epsilon}_{1} & \dot{\boldsymbol{\epsilon}}_{1} & \boldsymbol{\epsilon}_{2} & \dot{\boldsymbol{\epsilon}}_{2} & \boldsymbol{\epsilon}_{3} & \dot{\boldsymbol{\epsilon}}_{3}
\end{array}\right]^{T} \\
\mathbf{u} & =\left[\begin{array}{lllllll}
m_{x} & m_{y} & m_{z} & \dot{h}_{w x} & \dot{h}_{w y} & \dot{h}_{w z}
\end{array}\right]^{T}
\end{aligned}
$$

The system matrix $\mathbf{A}$ and the actuator matrix $\mathbf{B}(t)$ are found by standard linearization techniques, and will not be given here. However, it should be noted that since the satellite orbit is circular, the system matrix $\mathbf{A}$ will be constant and time-invariant. On the other hand, the actuator matrix $\mathbf{B}(t)$ will be time-varying when magnetic torquers are used for actuation, due to the implications of the geomagnetic field of the Earth and its aperiodic and time-varying nature. Details of the system linearization can be found in (Kristiansen, 2000).

\subsection{Linear Quadratic ( $L Q)$ controller}

For the purpose of controlling the attitude of the satellite in orbit, an LQ optimal controller will be used. By minimization of a quadratic performance index

$$
\min J=\frac{1}{2} \int_{0}^{t}\left(\tilde{\mathbf{x}}^{T}(\sigma) \mathbf{Q} \tilde{\mathbf{x}}(\sigma)+\mathbf{u}^{T}(\sigma) \mathbf{P u}(\sigma)\right) \mathrm{d} \sigma
$$

where $\tilde{\mathbf{x}}$ is the system error vector, $\mathbf{P}>0$ and $\mathbf{Q} \geq 0$ are constant weighting matrices, the optimal control law can according to Athans \& Falb (1966) be obtained by utilizing the feedback from the state $\mathbf{x}(t)$ and the feed forward from the desired state $\mathbf{x}_{d}(\mathrm{t})$ as

$$
\mathbf{u}(\mathbf{t})=-\mathbf{P}^{-1}(t) \mathbf{B}^{T}(t) \mathbf{p}(t)
$$

Furthermore, $\mathbf{p}$ can be expressed as the linear combination

$$
\mathbf{p}(t)=\mathbf{R}(t) \mathbf{x}(t)+\mathbf{h}_{\mathbf{l}}(t)
$$


where $\mathbf{R}(t)$ must be solved from the differential Ricatti equation

$$
\dot{\mathbf{R}}+\mathbf{R A}+\mathbf{A}^{T} \mathbf{R}-\mathbf{R B P}^{-1} \mathbf{B}^{T} \mathbf{R}=-\mathbf{Q}
$$

and $\mathbf{h}_{\mathrm{J}}(t)$ is the solution of

$$
\dot{\mathbf{h}}_{1}+\left(\mathbf{A}-\mathbf{B} \mathbf{P}^{-1} \mathbf{B}^{T} \mathbf{R}\right) \mathbf{h}_{1}=\mathbf{Q} \mathbf{x}_{d}
$$

The boundary conditions for (21) and (22) are given as

$$
\mathbf{h}_{1}(T)=\mathbf{0} \quad \text { and } \quad \mathbf{R}(T)=\mathbf{0}
$$

Hence, the differential equations for $\mathbf{h}_{1}(t)$ and $\mathbf{R}(t)$ can be solved for all $t \in[0, T]$ by backward integration. The weighting matrices $\mathbf{P}$ and $\mathbf{Q}$ are defined as

$$
\begin{aligned}
& \mathbf{P}=\operatorname{diag}\left(\left[p_{1}, p_{2}, \ldots, p_{n}\right]\right) \\
& \mathbf{Q}=\operatorname{diag}\left(\left[q_{1}, q_{2}, \ldots, q_{n}\right]\right)
\end{aligned}
$$

and the individual weights are chosen according to (Balchen \& Mummé 1988) as

$$
p_{i}=\frac{1}{\left(\Delta x_{i}\right)^{2}} \text { and } \quad q_{i}=\frac{1}{\left(\Delta u_{i}\right)^{2}}
$$

where $\Delta x_{i}$ and $\Delta u_{i}$ are the nominally acceptable variation in the states and actuator torques, respectively.

\section{Controllability}

For the purpose of evaluating the controllability of the different actuator configurations, a gramian-based analysis is performed in the following, based on controllability definitions given in Antsaklis \& Michel (1997). A solution for the linearized satellite model given in (20) can be expressed as

$$
\mathbf{x}(t)=\boldsymbol{\Phi}\left(t, t_{0}\right) \mathbf{x}_{0}+\int_{t_{0}}^{t} \boldsymbol{\Phi}(t, \tau) \mathbf{B}(\tau) \mathbf{u}(\tau) d \tau
$$

where $\mathbf{x}_{0}=\mathbf{x}\left(t_{0}\right)$ is the initial state, $t \in[0, T]$, and $\boldsymbol{\Phi}\left(t, t_{0}\right)$ denotes the state transition matrix for the transition from state $x_{0}$ at time $t_{0}$ to state $x$ at time $t$. For a linear system with a time-invariant system matrix $\mathbf{A}$ as in (20), the state transition matrix can be written as

$$
\Phi\left(t, t_{0}\right)=e^{\mathbf{A}\left(t-t_{0}\right)}
$$

The system (20) is said to be state controllable at time $t_{0}$, if there exists a finite time $t_{1}>t_{0}$ such that for any $\mathbf{x}\left(t_{0}\right)$ there exists an input $\mathbf{u}(t)$ that will transfer the state $\mathbf{x}\left(t_{0}\right)$ to the state $\mathbf{x}\left(t_{1}\right)$. Otherwise the state equation is said to be uncontrollable at time $t_{0}$ Wie (1998). The trajectory taken by the state is not specified, since any trajectory will do. It should also be noted that there is no constraint on the control inputs and the magnitude of these. Furthermore, the controllability gramian $\mathbf{W}_{c}\left(t_{0}, t_{1}\right)$ of the system (20) is the $n \times n$ matrix

$$
\mathbf{W}_{c} \triangleq \int_{t_{0}}^{t_{1}} \boldsymbol{\Phi}\left(t_{0}, \tau\right) \mathbf{B}(\tau) \mathbf{B}^{T}(\tau) \boldsymbol{\Phi}^{T}\left(t_{0}, \tau\right) d \tau
$$

The system is said to be controllable if the matrix $\mathbf{W}_{c}\left(t_{0}, t_{1}\right)$ is positive definite,

$$
\mathbf{W}_{c}\left(t_{0}, t_{1}\right)>0
$$


The natural selection of the time period $t_{1}$ in this analysis is the time for the satellite to complete one orbit, which can be calculated as the orbit angle in one orbit period divided by the orbit angular velocity,

$$
t_{1}=\frac{2 \pi}{\omega_{o}} \approx 5793 \mathrm{~s}
$$

and the starting time is set to zero, $t_{0}=0$. A numerical Runge-Kutta method of order four is used for integration of the gramian. To investigate the positive definiteness of the resulting matrices, Sylvester's theorem of eigenvalues Strang (1988) is used, which states that since $\mathbf{W}_{c}\left(t_{0}, t_{1}\right)$ is symmetric, a necessary and sufficient condition for positive definiteness is that all the matrix eigenvalues are strictly positive. It is therefore necessary to ensure that the smallest eigenvalue of $\mathbf{W}_{c}\left(t_{0}, t_{1}\right)$ is larger than zero,

$$
\lambda_{\min }\left(\mathbf{W}_{c}\left(t_{0}, t_{1}\right)\right)>0
$$

Because of the difference in actuator torques from the magnetic coils and the reaction wheels, the actuator matrix in the linearized system should be normalized. The magnetic coils delivers a maximum torque of $8 \mathrm{Am}^{2}$, while the wheels deliver $0.1-0.01 \mathrm{Nm}$. In this analysis a maximum wheel torque of $0.01 \mathrm{Nm}$ is considered, and the actuator term in (20) can be expressed as

$$
\mathbf{B}(t) \mathbf{u}=\mathbf{B}(t) \mathbf{N}^{-1} \mathbf{N u}=\mathbf{B}(t) \mathbf{N}^{-1} \mathbf{u}_{n o r m}(t)
$$

where $\mathbf{u}_{\text {norm }}=\mathbf{N u}$ is the normalized actuator vector where all the torques vary from -1 to 1 . The scaling matrix $\mathbf{N}$ can now be obtained as

$$
\mathbf{N}=\operatorname{diag}\left(\frac{1}{8}, \frac{1}{8}, \frac{1}{8}, 100,100,100\right)
$$

and its inverse is thus

$$
\mathbf{N}^{-1}=\operatorname{diag}\left(8,8,8, \frac{1}{100}, \frac{1}{100}, \frac{1}{100}\right)
$$

inserting these normalization terms into (20) leaves the normalized system as

$$
\dot{\mathbf{x}}(t)-\mathbf{A} \mathbf{x}(t)+\mathbf{B}(t) \mathbf{N}^{-1} \mathbf{u}_{\text {norm }}(t)
$$

The results of the theoretical analysis are presented in Table 1. The first obvious, and expected, result in Table 1 is that the satellite controllability in general improves radically for every reaction wheel added. In addition, the expected performance improvement when using three reaction wheels instead of magnetic torquers is shown.

In regard to optimal locations for the reaction wheels, it should be noted that a reaction wheel on the pitch axis $y$ will not provide the same improvement as a reaction wheel on the roll axis $x$ or the yaw axis $z$. Placing a wheel on the latter two axes will result in approximately the same improvement. A slight difference between the effect of placing a reaction wheel on the roll axis $x$ or yaw axis $z$ is also seen in Table 1 . Since both of these axes are coupled by the actuator torque when using magnetic actuation (actuated by same torquer), it is to be expected that placing a wheel on either of these axes results in an improvement. Also, since the moments of inertia about these axes are very different when the gravity boom is deployed, it is expected that a reaction wheel, which provides more torque than a magnetic torquer, will be of more use on the roll axis $x$, since this axis has a larger moment of inertia and thus needs more torque for control. 
Table 1. Smallest eigenvalues of the controllability gramian in the different configurations

\begin{tabular}{ll}
\hline Actuator configuration & $\lambda_{\min }\left(\mathbf{W}_{c}\left(t_{0}, t_{1}\right)\right)$ \\
\hline $\begin{array}{l}\text { No reaction wheels } \\
\text { Magnetic coils only }\end{array}$ & $3.6686 \cdot 10^{-10}$ \\
One reaction wheel & \\
Axis $x$ & $1.3410 \cdot 10^{-9}$ \\
Axis $y$ & $3.6813 \cdot 10^{-10}$ \\
Axis $z$ & $1.3193 \cdot 10^{-9}$ \\
Two reaction wheels & \\
Axes $x$ and $y$ & $1.5169 \cdot 10^{-6}$ \\
Axes $y$ and $z$ & $1.0002 \cdot 10^{-7}$ \\
Axes $x$ and $z$ & $1.3420 \cdot 10^{-9}$ \\
Three reaction wheels & \\
Axes $x, y$, and $z$ & $3.3410 \cdot 10^{-3}$ \\
\hline
\end{tabular}

\section{Simulations}

\subsection{Satellite data}

In the following section, simulation results for different actuator configurations will be presented. The satellite, including the gravity boom, is assumed to be a rigid body, with moments of inertia given as

$$
\mathbf{J}=\operatorname{diag}\left\{\begin{array}{lll}
178 & 181 & 4.3
\end{array} \mathrm{kgm}^{2}\right.
$$

with the gravity boom deployed and

$$
\mathbf{J}=\operatorname{diag}\left\{\begin{array}{lll}
4 & 4 & 3
\end{array}\right\} \mathrm{kgm}^{2}
$$

with the boom stowed. The magnetic torquers on the satellite are assumed to produce a maximum dipole moment of $8 \mathrm{Am}^{2}$, and the reaction wheels are taken to have a maximum speed of $6000 \mathrm{rpm}$, and a maximum output torque of $0.01 \mathrm{Nm}$. The satellite is assumed to rotate about the Earth in a $600 \mathrm{~km}$ circular polar orbit, and the satellite insertion point in the simulations is above the Equator. The magnetic field of the Earth is approximated with spherical harmonics of order 10 and degree 10 . The satellite attitude is simulated using quaternions, but the results are converted to Euler angles before they are presented, to improve viewing. The satellite system was simulated with an initial deviation from the equilibrium point, which would be the case in for example

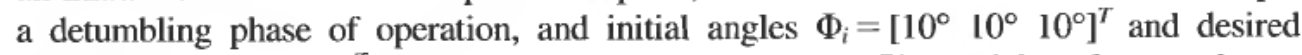
angles $\Phi_{d}=\left[\begin{array}{lll}0^{\circ} & 0^{\circ} & 0^{\circ}\end{array}\right]^{T}$ were used in all the simulations. The initial angles are chosen equal to visualize the difference of performance in the three axes. They are also chosen relatively close to the equilibrium point, which is the usual working area for a satellite with a gravity boom. Also, it is easier to recognize the implications of the magnetic field when the deviations are small. The LQ controller parameters are tuned to give the best possible performance for each actuator configuration. The desired attitude accuracy for the satellite is within $\pm 0.5^{\circ}$ in all axes. A possible scheme for this is a surveillance satellite with a payload for Earth imaging applications. 

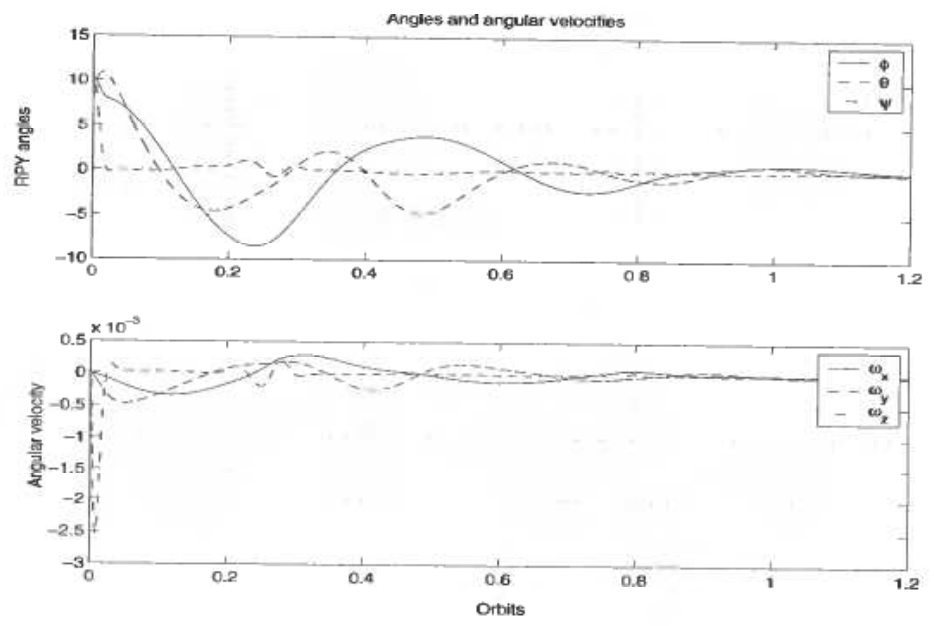

Figure 2. Step response of the satellite actuated by three orthogonal magnetic torquers.

\subsection{Results}

5.2.1. Three magnetic torquers only. The result from simulation of the satellite actuated by three magnetic torquers is shown in Figure 2. In addition to the magnetic torquers, the gravity boom was deployed and used for passive stabilization. The satellite settled within $\pm 0.5^{\circ}$ after approximately 1.2 orbit periods, and the total power consumption for the entire maneuver was $7.7 \cdot 10^{-5} \mathrm{~W}$. The peak in angular velocity, and the settling time for the different axes, together with the slight deviation in yaw angle after about one quarter of the orbit should also be observed. The reason for the rapid response in the yaw axis of the satellite, together with the loss of controllability in the same axis after about one quarter of the orbit, stems from the dependency on the magnetic field. The yaw axis is controllable when the satellite passes by the equator, but not when it passes by the poles.

5.2.2. One reaction wheel. Figure 3 shows the results of actuating the satellite with three orthogonal magnetic torquers and one reaction wheel, which was located on the body roll axis. In addition, the gravity boom was deployed for passive stabilization. Note that the scale on the time-axis is stretched relative to Figure 2. There was no problem for this configuration to achieve the desired accuracy after one orbit period, and the improvement in setuling time for the roll axis was good. However, this configuration added a great deal of overshoot for the first turn on the yaw axis, and the decreased controllability of the pitch axis should be observed, together with the slight deviation in the yaw axis after approximately one quarter of the orbit, which occur when the magnitude of the magnetic field vector orthogonal to the torquer on the pitch axis is small. The angular velocities of the satellite also increased due to the adding of the wheel, and the power consumption was $6.8 \cdot 10^{-4} \mathrm{~W}$.

With the reaction wheel moved from the roll axis to the pitch axis, the simulation results were as shown in Figure 4. The figure shows that less than $\pm 0.5^{\circ}$ attitude deviation was achieved within one orbit, but with a slight increase of oscillations before settling, especially in the roll motion. The total power consumption also increased to $1.2 \cdot 10^{-3} \mathrm{~W}$. 

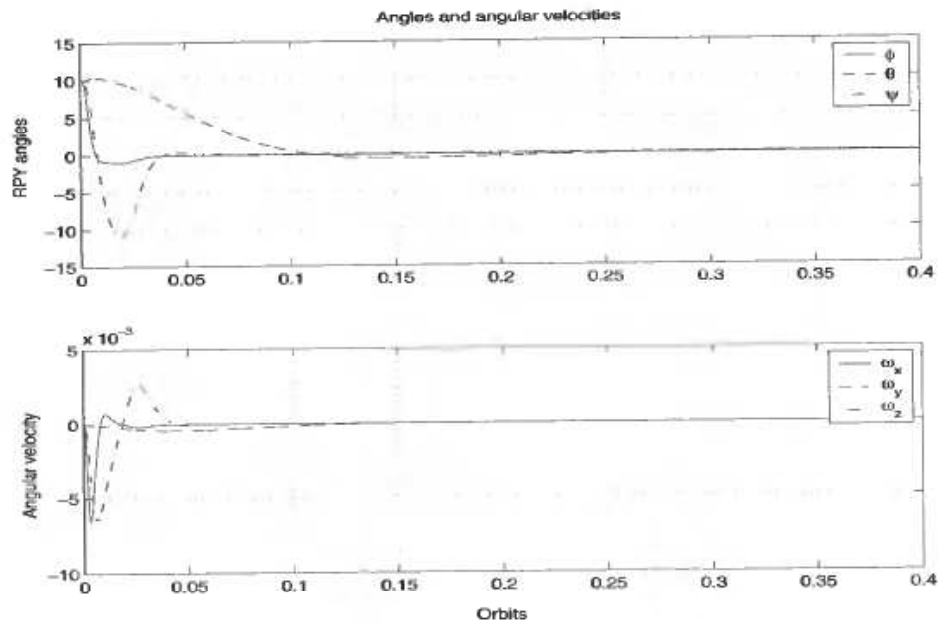

Figure 3. Step response of the satellite actuated by three orthogonal magnetic torquers and a reaction wheel on the roll axis.
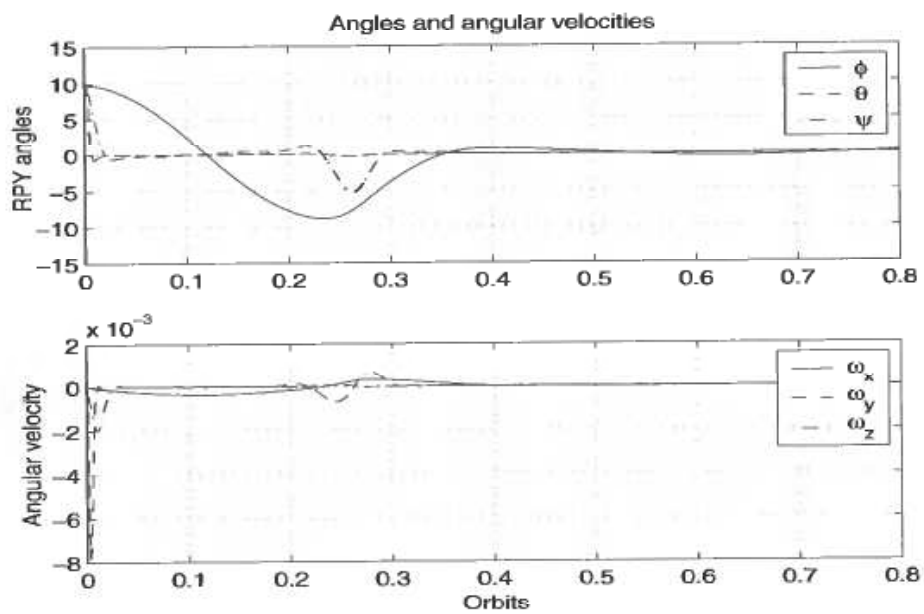

Figure 4. Step response of the satellite actuated by three orthogonal magnetic torquers and a reaction wheel on the pitch axis.

Finally, placing a reaction wheel on the yaw axis results as illustrated in Figure 5 . This configuration should according to the theoretical analysis have approximately the same controllability as the configuration with a reaction wheel placed on the roll axis. The satellite oscillated a great deal, but remained within $\pm 30^{\circ}$ of the equilibrium point. This was unexpected in view of the gramian analysis, and the likely reason for this is the magnitude of the output torque of the wheel. Figure 6 shows the step response of the satellite with the same actuator configuration, but with a lower output torque on the yaw reaction wheel. The satellite stopped oscillating due to the decreased output torque, but now suffered from poor controllability. This is a trend in all the configurations involving a reaction wheel on the yaw axis, and further results for such configurations will therefore not be presented here. Details about these results can be found in (Kristiansen, 2000). 

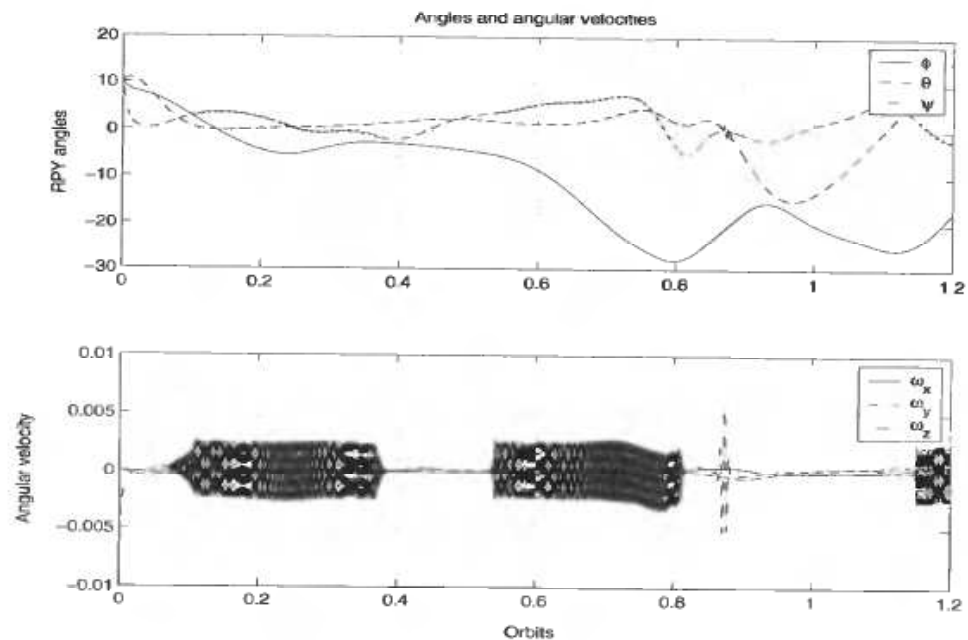

Figure 5. Step response of the satellite actuated by three orthogonal magnetic torquers and a reaction wheel on the yaw axis.
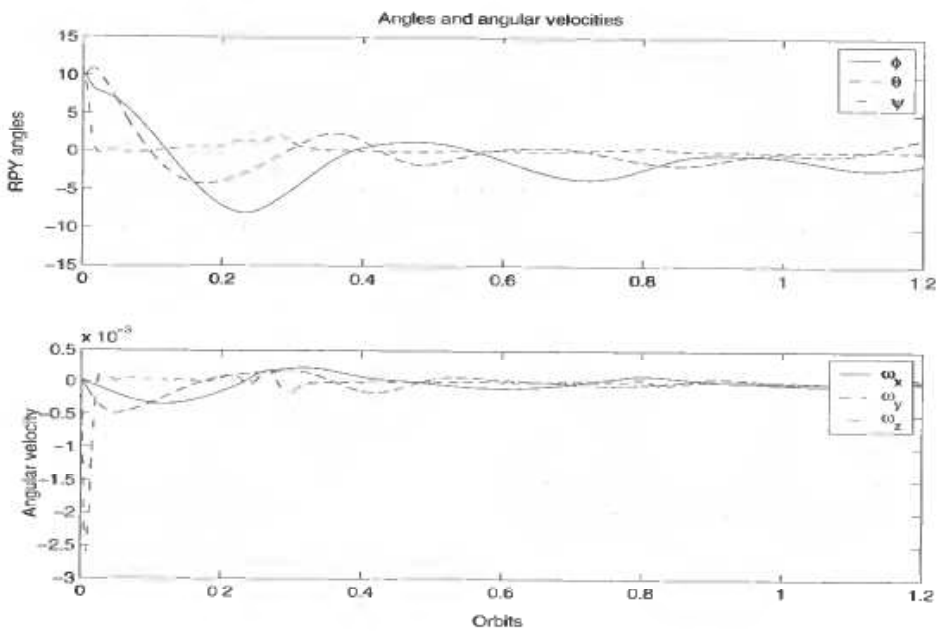

Figure 6. Step response of the satellite actuated by three orthogonal magnetic torquers and a reaction wheel on the yaw axis with decreased output torque.

5.2.3. Two reaction wheels. Combining the previous two actuator configurations, leaves a satellite actuated by three magnetic torquers and two reaction wheels, located on the body roll and pitch axes. The gravity boom was also deployed to passively stabilize the satellite. The result of this configuration is presented in Figure 7. Again, the denomination on the time-axis of the figure should be noticed. The overall response improved significantly, and the satellite settled within 0.15 orbits. The total amount of consumed power during the maneuver was $1.7 \cdot 10^{-3} \mathrm{~W}$.

5.2.4. Three reaction wheels. When three reaction wheels are used as actuators on the satellite, the magnetic torquers can be neglected regarding active attitude control of the satellite, due to their low thrust torque compared to the reaction wheels. It should be noticed that the gravity boom was removed in this configuration. This is done based on 

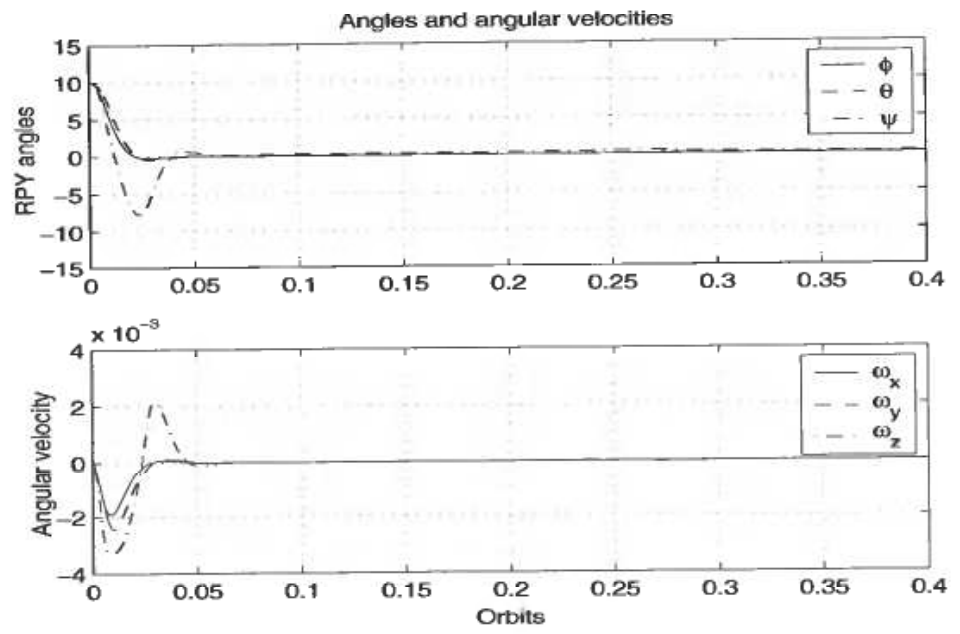

Figure 7. Step response of the satellite actuated by three orthogonal magnetic torquers and a reaction wheels on the roll and pitch axes.
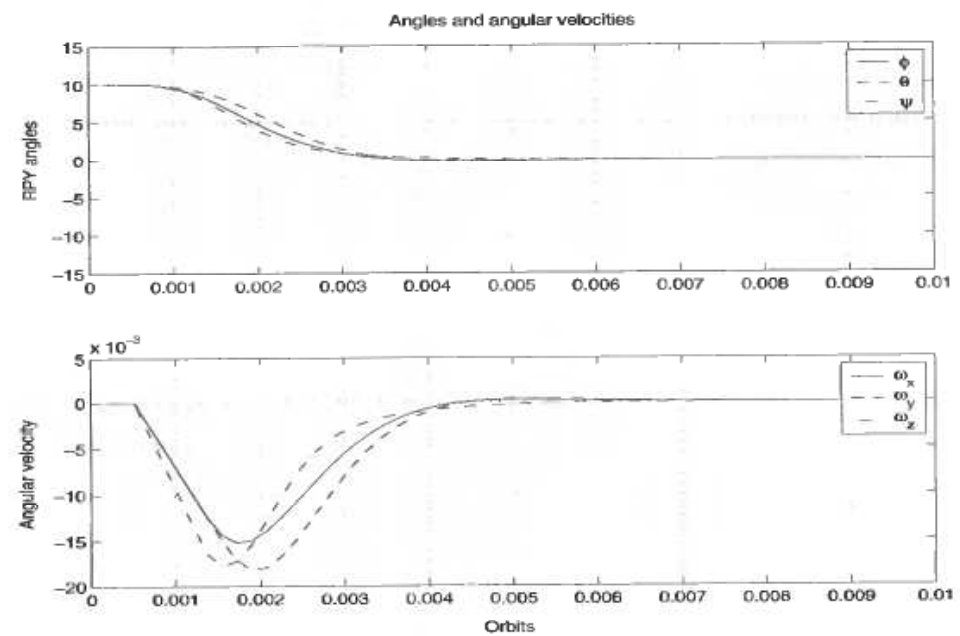

Figure 8. Step response of the satellite without the gravity boom, actuated by three orthogonal reaction wheels.

a mission viewpoint, since a satellite controlled by three reaction wheels will be more vulnerable to wheel saturation when a gravity boom is used, since it requires more torque to rotate the satellite and to keep it in orientations where the boom is not parallel to the local vertical. For satellite reorientations from an initial angle deviation towards the equilibrium point, this will have small impact on the controller performance and power consumption, since the gravity torque from the boom is negligible compared to the torque provided by the reaction wheels. The simulation result is presented in Figure 8. This is the configuration with the most satisfactory controllability result in the theoretical analysis, and the improvements can clearly be seen in the figure. The satellite settled within the angular limitations after 0.005 orbit periods, which corresponds to approximately thirty seconds. In addition, no overshoot is observed, and the satellite body frame is equal to the orbit frame. This is a very satisfactory result, and substantiates the result found in the gramian analysis in Table 1. On the other hand, the increase in angular 

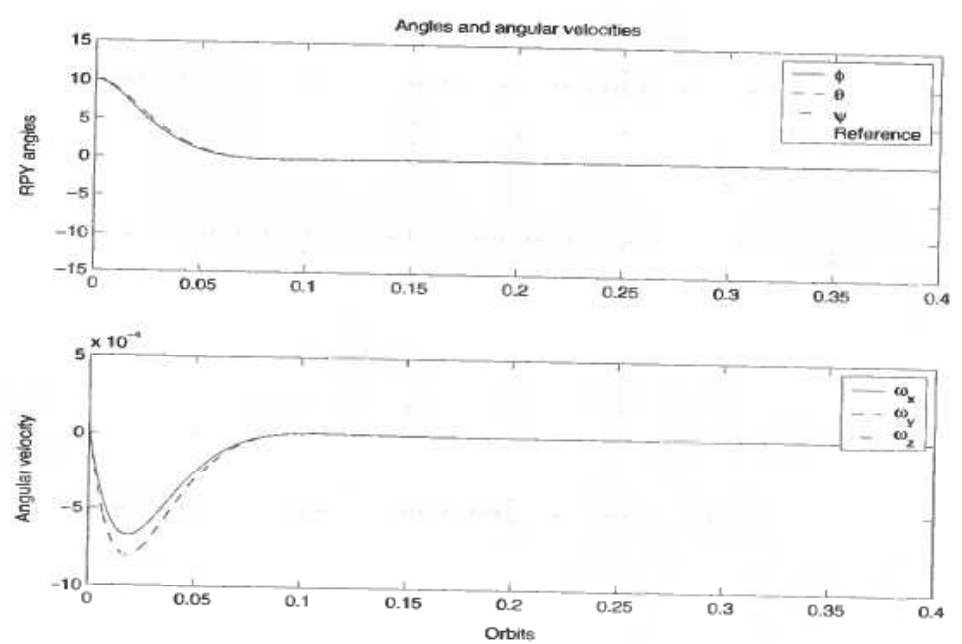

Figure 9. Response of the satellite actuated by three orthogonal reaction wheels. tracking a reference trajectory generated by a second order filter.

velocity in all axes should be observed, which was introduced by the removal of the gravity boom. In addition, the gravitational torque on the satellite has been greatly reduced, and the satellite will thus be able to rotate faster. However, it also entail degraded robustness towards external disturbances, since the gravity gradient stabilization has been removed. The reaction wheels will introduce some stabilizing effects when they are rotating, but not in the same magnitude as the gravity gradient. Still, the satellite will be robust towards external disturbances due to the increase in available torque.

The total power consumption of the maneuver was $2.9 \cdot 10^{-3} \mathrm{~W}$, a large amount relative to the $7.7 \cdot 10^{-5} \mathrm{~W}$ consumed for the same maneuver when magnetic torquers were used for actuation. Also, high angular velocities on the satellite can sometimes be a problem for onboard equipment, i.e. attitude determination sensors. To remedy this, a reference trajectory can be generated that slows down the satcllite in slew maneuvers. This can be achieved by introducing a second order filter with a cross-over frequency $\omega_{n}=0.01$, and a relative damping factor $\zeta=0.8$ to generate the reference trajectory. The resulting behavior of the satellite is shown in Figure 9. The satellite followed the reference trajectory, and the angular velocities were kept low. In addition, monitoring the power consumption actually shows that the control system consumes $5.7538 \cdot 10^{-6} \mathrm{~W}$ before settling, which is lower than the power consumption of three magnetic torquers in the same step.

\section{Conclusion}

This paper presents results from the analysis on controllability of a mini satellite by performing numerical calculations based in the controllability gramian. The analysis is performed by numerically calculating the gramian integral, and then studying the eigenvalues of the resulting matrix. The smallest eigenvalues of the gramians found for different actuator configurations have been presented. The results of this analysis have been supported by simulation results. It was found that the performance of an attitude control system consisting of three orthogonal magnetic torquers and a gravity boom is 
significantly improved by adding one reaction wheel. The improvement is actually almost $600 \%$, and caused the satellite to settle after 0.2 orbit periods rather than 1.2 orbit periods. Including even one more reaction wheel in the optimal axis will also result in an improved performance, but not of the same magnitude. When three reaction wheels were used for actuation, the satellite settled from initial angles in 0.005 orbit periods. Regarding the location of the reaction wheels, comparison of the simulation results indicates that the roll axis is the optimal location for one reaction wheel on this satellite. Adding a reaction wheel in the pitch axis does not improve satellite controllability to the same extent as using a wheel in the roll axis.

The problem with the restricted amount of available energy in the satellite will be reduced when using magnetic actuation, compared to actuation by reaction wheels. According to the simulation results, three reaction wheels will consume approximately 32 times more power than three magnetic torquers. However, when using magnetic actuation, the satellite controller has to be used actively over large periods of time to rotate the satellite to desired orientations. When reaction wheels are providing the torque, the same change of attitude will requirc less time, and the satellite will be idle in some time periods. In these periods the satellite could be rotated to get an optimal angle for the solar arrays towards the sun, and in that way compensate for its relatively large use of energy for stabilization. Simulations also showed that introduction of smooth reference trajectories instead of abrupt step changes reduced the power consumption. In fact, using this approach a satellite actuated by three reaction wheels consumed 30 times less power than a satellite with three magnetic torquers, but still the response of the satellite was satisfactory. This is a good argument for using reaction wheels together with a reference model for actuation. In this paper, the reference model is used only as a means to lower the power consumption when reaction wheels are used for actuation. However, it will probably have a positive effect for configurations consisting of magnetic torquers also. A satellite using magnetic actuation will have available torque about a given axis in some periods of time. If the attitude errors are large, the actuators will be used extensively to rotate the satellite, and thereby increasing the angular velocity. If the satellite looses its controllability when the angular velocities are high, it will not be able to decelerate. If a reference model is used, there will not be large attitude errors and hence, the angular velocities of the satellite are kept low.

Even though the results described in this paper are based on a specific satellite, they will extend to a more general result for small satellites. The choice of actuator configurations is typically done based on a tradeoff between control performance and power consumption. For a low powered satellite with low accuracy requirements, the typical configuration consists of magnetic torquers and a gravity boom. However, with higher accuracy requirements, the solution is often to equip the satellite with three reaction wheels, remove the gravity boom, and keep the magnetic torquers for momentum dumping. However, as this study shows, this is a drastic solution, as adding only one rcaction wheel to the satellite will significantly improve the performance of the controller. On the other hand, magnetic torquers are often used on satellites among other reasons because of their low power consumption. However, this study shows that the relatively higher power consumption for the reaction wheels can be significantly decreased by introducing reference trajectories in the control loop. The result of this is that the power consumption is not longer an issue when debating the use of reaction wheels versus magnetic torquers. 


\section{References}

ANTSAKLIS, P. J. \& Michel, A. N. (1997). Linear Systems, McGraw-Hill Electrical and Computer Engineering Series, McGraw-Hill, New York, USA.

Athans, M. \& Falb, P. L. (1966). Optimal Control, McGraw-Hill Book Company, New York.

BAK, T., BlankE, M. \& WiśNIEWSKI, R. (1999). Flight results and lessons learned from the Ørsted attitude control system, Proceedings of the 4th ESA International Conference on Spacecraft Guidance, Navigation and Control Systems, ESTEC, Noordwijk, The Netherlands.

BAK, T. (2000). Spacecraft Attitude Determination-a Magnetometer Approach. Ph.D. thesis, Aalborg University, Aalborg, Denmark.

Balchen, J. G. \& Mummé, K. I. (1988). Process Control-Structures and Applications, Van Nostrand Reinhold, New York.

BøGH, S. A., WIŚNIEWSKI, R. \& BAK, T. (1997). Autonomous Attitude Control System for the Ørsted Satellite, Proceedings of the IFAC Workshop on Control of Small Spacecraft, Breckenbridge, USA.

CAmpbell, W. H. (1997). An Introduction to Geomagnetic Fields. Cambridge University Press, Cambridge, UK.

Egeland, O. \& GRAVDahl, J. T. (2002). Modeling and Simulation for Automatic Control, Marine Cybernetics, Trondheim, Norway.

Gravdahl, J., Eide, E., Skavhaug, A.., Fauske, K. M., Svartvert, K. \& Indergaard, F. M. (2003). Three Axis Attitude Determination and Control System for a Picosatellite: Design and Implementation, Proceedings of the 54th International Astronautical Congress, Bremen, Germany.

Kaplan, M. H. (1976). Modern Spacecraft Dynamics and Control, John Wiley \& Sons, New York, USA.

Kristiansen, R. (2000). Attitude Control of a Microsatellite, Master's thesis. Department of Engineering Cybernetics, Norwegian University of Science and Technology, Trondheim, Norway.

Marshall, S. V. \& Skitek, G. G. (1990). Electromagnetic Concepts and Applications, PrenticeHall International Inc, New Jersey, USA.

Rise, Å.-R., Samuelsen, B., Sokolova, N., Cederblad, H., Fasseland, J., Nordin, C., OtTerstad, J., Fauske, K., Eriksen. O., Indergaard, F. M., Svartveit, K., Furebotten, P., Sather, E. \& EIDE, E. (2003). Ncube: The First Norwegian Student Satellite, Proceedings of The 17th AIAA/USU Conference on Small Satellites, Logan, Utah, USA.

SIDI, M. J. (1997). Spacecraft Dynamics and Control. Cambridge University Press, New York, USA.

STEYN, W. H. \& HASHidA, Y. (1999). In-orbit Attitude and Orbit Control commissioning of UoSAT-12, Proceedings of the 4th ESA International Conference on Spacecraft Guidance, Navigation and Control Systems, ESTEC, Noordwijk, The Netherlands.

Strang, G. (1988). Linear Algebra and its Applications, Harcourt Brace Jovanovich College Publishers, Orlando, Florida, USA.

VON SCHÉElE, F. (1996). The Swedish Odin Satellite to Eye Heaven and Earth, Proceedings of the 47th International Astronautical Congress, Beijing, China,

WerTZ, J. R. (1978). Spacecraft Attitude Determination and Control, Kluwer Academic Publishers, London, UK.

WIE, B. (1998). Space Vehicle Dynamics and Control, AlAA Education Series, American Institute of Aeronautics and Astronautics, Inc., Reston, Virginia, USA. 